\title{
Robust Parameter Identification for Cardiac diagnosis in critical care using minimal data sets
}

C.E. Hann ${ }^{1}$, J.G. Chase ${ }^{1}$, T. Desaive ${ }^{3}$, C.F. Froissart ${ }^{2}$, J. Revie ${ }^{1}$, D. Stevenson ${ }^{1}$, B. Lambermont $^{3}$, A. Ghuysen ${ }^{3}$, P. Kolh ${ }^{3}$ and G.M. Shaw ${ }^{4}$

${ }^{1}$ Department of Mechanical Engineering, Centre for Bio-Engineering, University of Canterbury, Christchurch, New Zealand Email: Chris.Hann@canterbury.ac.nz

${ }^{2}$ Université de Technologie de Belfort-Montbéliard, France

${ }^{3}$ Hemodynamics Research Laboratory, University of Liege, Belgium

${ }^{4}$ Department of Intensive Care, Christchurch Hospital, Christchurch, New Zealand

For the definitive version of this article, see:

Hann, C.E., Chase, J.G., Desaive, T., Froissart, C.B., Revie, J., Stevenson, D., Lambermont, B., Ghuysen, A., Kolh, P. and Shaw, G.M. (2010) Unique parameter identification for cardiac diagnosis in critical care using minimal data sets. Computer Methods and Programs in Biomedicine, 99(1), 75-87. http://dx.doi.org/10.1016/j.cmpb.2010.01.002 


\begin{abstract}
Lumped parameter approaches for modeling the cardiovascular system typically have many parameters of which many are not identifiable. This paper considers the modeling and the parameter identification simultaneously, and creates models that are one to one with the measurements. That is, every input parameter into the model is uniquely optimized to capture the clinical data and no parameters are set at population values. In addition, simplified sub-structures of the six chamber model are created to provide very fast and accurate parameter identification from arbitrary starting points and with no prior knowledge on the parameters. Furthermore, by utilizing continuous information from the arterial/pulmonary pressure waveforms and the end-diastolic time, it is shown that only the stroke volumes of the ventricles are required for adequate cardiac diagnosis. This reduced data set is more practical for an intensive care unit as the maximum and minimum volumes are no longer needed, which was a requirement in prior work. The simplified models can also act as a bridge to identifying more sophisticated cardiac models, by providing a generating set of waveforms that the complex models can match to. Most importantly, this approach does not have any predefined assumptions on patient dynamics other than the basic model structure, and is thus suitable for improving cardiovascular management in critical care by optimizing therapy for individual patients.
\end{abstract}

Keywords: model-based cardiac diagnosis, cardiovascular system, integral-based parameter identification, pressure waveform, ECG, Intensive Care Unit

\title{
1. Introduction
}


In critical care, cardiovascular dysfunction can be easily misdiagnosed due to the array of sometimes conflicting data [1-3]. It is also a major cause of increased length of stay and death $[4,5]$. Demand for critical care is also growing dramatically severely affecting healthcare delivery [6-8]. The overall goal of this research is to use computational cardiac models to better aggregate available clinical data in an intensive care unit (ICU) into a more readily understood physiological context for clinicians. The computational models can be used to reveal dynamics and interactions non-obviously hidden in the data, enabling simpler and more robust diagnosis.

A major difficulty faced with cardiovascular modelling is the level of detail these models typically include. For example multi-scale modelling approaches utilizing finite elements have successfully explained complex behaviour of the heart [9-11]. However, a large gap exists between the computational results of these detailed models and clinical utility.

Lumped parameter models (LPM) are a common approach to minimizing complexity in the cardiovascular system [12-16] but there are still many parameters involved. Thus typically, only small subsets of the parameter set can be identified (e.g. [14]). As a result, a majority of the parameter set has to be fixed at population values, which demands prior knowledge on the state of patients and a homogeneity between patients that may not exist.

In critical care, a patient's condition can change rapidly and therefore any preassumption on parameters may jeopardise accurate diagnosis. Furthermore, the more complex a LPM becomes, the larger the set of unidentifiable parameters, and the greater the number of dynamics that may differ from the actual. Increased computational requirements with increasing parameters or model complexity will also limit real-time patient specific application at the bedside.

This paper presents a different approach, by first developing simplified, fully identifiable, patient specific models, that are based around the clinical data available in an ICU. These models can serve as a bridge to identify more complicated and 
physiologically accurate models as required to predict the observed patient hemodynamic responses. In the simplified models, patient specific dynamics are only considered if they can be uniquely identified from the given data. Due to the simplified structure of these models, it is then possible to analyze individual geometric effects of given input parameters on the output. This information will lead to the minimal set of features in the outputs, that are required for adequate cardiac diagnosis.

Note that the word identifiability referred to here is "practical identifiability" with respect to noise and modelling error, which can currently only be tested numerically $[17,18]$. General identifiability theory $[19,20]$ refers to the ideal case of perfect knowledge of the system and measurements, which does not guarantee that parameters can be identified uniquely in practice. Hence, this study views identifiability from the perspective of the final application.

The starting baseline model structure considered is a six chamber cardiovascular model including ventricular interaction and inertial effects that has been previously developed [16] and validated in clinical animal trials [21-24]. However, note that the approach is general and could be applied to any cardiac model structure.

A new concept developed in this paper matches simplified CVS model outputs to continuous information of arterial/pulmonary pressures and the end-diastolic time from an ECG or the "a wave” timing from the pulmonary pressure waveform. Adding continuous pressure waveforms and end-diastolic timing to current clinical data sets is shown to increase the diagnostic ability of the model and enable a more minimal data set that doesn't require maximum and minimum volume measurements. Hence, this approach adds a simple and easy measurement to remove the need for a more invasive, difficult and noisy measurement.

New methods are rigorously tested in simulation with noise corrupted measurements and modelling error to prove robustness. Finally, animal data is used to demonstrate the clinical potential of these methods.

\section{Methodology}




\subsection{Cardiac model}

The cardiovascular system model used in this paper consists of six elastic chambers, as shown in Figure 1. First developed in [16], it has been validated clinically [21-24]. All the input parameters for a healthy human baseline state are defined in Table 1. The output parameters are shown in Table 2 [25].

For simplicity, only the differential equations associated with the left ventricle are shown here, where [16] has a description of the full model.

$$
\begin{gathered}
L_{m t} \dot{Q}_{m t}=H\left(H\left(P_{p v}-P_{l v}\right)+H\left(Q_{m t}\right)-0.5\right)\left(P_{p v}-P_{l v}-R_{m t} Q_{m t}\right) \\
L_{a v} \dot{Q}_{a v}=H\left(H\left(P_{l v}-P_{a o}\right)+H\left(Q_{a v}\right)-0.5\right)\left(P_{l v}-P_{a o}-R_{a v} Q_{a v}\right) \\
\dot{V}_{l v}=H\left(Q_{m t}\right) Q_{m t}-H\left(Q_{a v}\right) Q_{a v} \\
\dot{V}_{a o}=H\left(Q_{a v}\right) Q_{a v}-Q_{s y s} \\
P_{a o}=E_{a o} V_{a o} \\
Q_{s y s}=\frac{P_{a o}-P_{v c}}{R_{s y s}} \\
P_{l v}=e(t) E_{e s, l v f}\left(V_{l v}-V_{s p t}\right)+(1-e(t)) P_{0, l v f}\left(e^{\lambda_{l v f}\left(V_{l v}-V_{s p t}\right)}-1\right)+P_{p e r i} \\
e(t)=e^{-80(t-p e r i o d / 2)^{2}} \\
H(K(t))=0, K(t) \leq 0 \\
=1, K(t)>0
\end{gathered}
$$

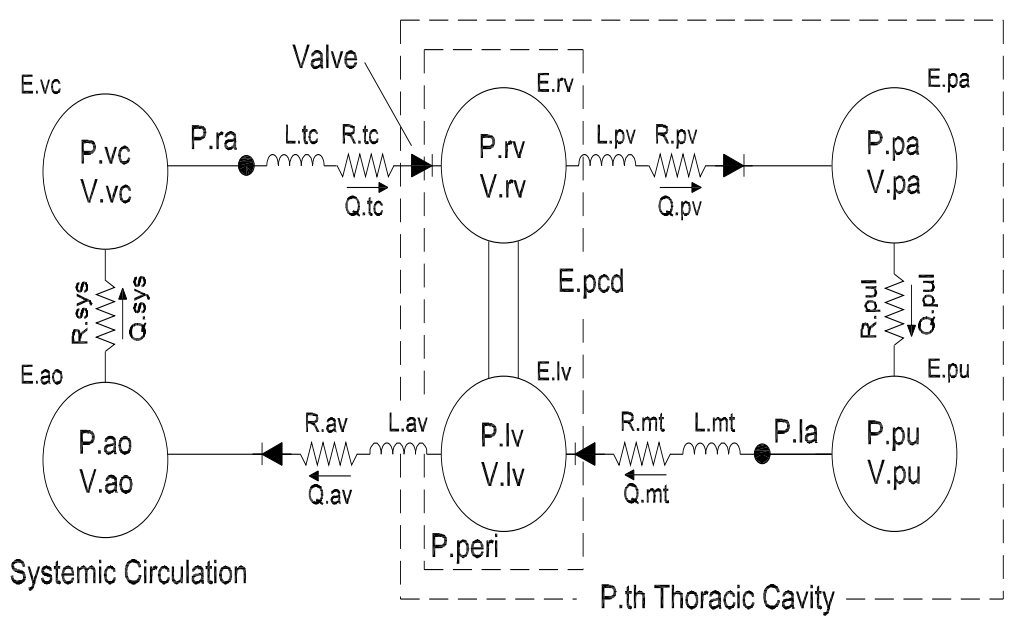

Figure 1: Six chamber CVS model with inertial effects and ventricular interaction 


\begin{tabular}{|c|c|c|c|}
\hline \multicolumn{2}{|c|}{ Parameters } & $\begin{array}{r}\text { Values } \\
06012\end{array}$ & Units \\
\hline$E_{a o}$ & Elastance of aorta & 0.6913 & $\mathrm{Hg} / \mathrm{ml}$ \\
\hline$R_{a v}$ & Resistance of aortic valve & 0.0180 & $\mathrm{mmHg} \mathrm{s} / \mathrm{ml}$ \\
\hline$L_{a v}$ & Inertance of aortic valve & $1.2189 \mathrm{e}-004$ & $\mathrm{mmHg} \mathrm{s}^{2} / \mathrm{ml}$ \\
\hline$E_{e s, l v f}$ & Elastance of left ventricle & 2.8798 & $\mathrm{Hg} / \mathrm{ml}$ \\
\hline$R_{m t}$ & Resistance of mitral valve & 0.0158 & $\mathrm{mmHg} \mathrm{s} / \mathrm{ml}$ \\
\hline$L_{m t}$ & Inertance of mitral valve & 7.6968e-005 & $\mathrm{mmHg} \mathrm{s} / \mathrm{ml}$ \\
\hline$R_{\text {sys }}$ & Systemic flow resistance & 1.0889 & $\mathrm{mmHg} \mathrm{s} / \mathrm{ml}$ \\
\hline$E_{p a}$ & Elastance of pulmonary artery & 0.3690 & $\mathrm{Hg} / \mathrm{ml}$ \\
\hline$E_{p u}$ & Elastance of pulmonary vein & 0.0073 & $\mathrm{Hg} / \mathrm{ml}$ \\
\hline$E_{e s, r v f}$ & Elastance of right ventricle & 0.5850 & $\mathrm{Hg} / \mathrm{ml}$ \\
\hline$E_{v c}$ & Elastance of vena cava & 0.0059 & $\mathrm{Hg} / \mathrm{ml}$ \\
\hline$R_{t c}$ & Resistance of tricuspid valve & 0.0237 & $\mathrm{mmHg} \mathrm{s} / \mathrm{ml}$ \\
\hline$R_{p v}$ & $\begin{array}{l}\begin{array}{l}\text { Resistance of pulmonary } \\
\text { valve }\end{array} \\
\end{array}$ & 0.0055 & $\mathrm{mmHg} \mathrm{s/ml}$ \\
\hline$R_{p u l}$ & Pulmonary flow resistance & 0.1552 & $\mathrm{mmHg} \mathrm{s} / \mathrm{ml}$ \\
\hline$E_{e s, s p t}$ & Elastance of the septum & 48.7540 & $\mathrm{Hg} / \mathrm{ml}$ \\
\hline \multicolumn{4}{|c|}{ Additional parameters } \\
\hline Period & Time of one heart beat & 0.75 & $\mathrm{~s}$ \\
\hline$P_{0, l v f}$ & $\begin{array}{l}\text { Defines gradient of EDPVR at } 0 \\
\text { pressure }\end{array}$ & 0.1203 & $\mathrm{mmHg}$ \\
\hline$P_{0, r v f}$ & $\begin{array}{l}\text { Defines gradient of EDPVR at } 0 \\
\text { pressure }\end{array}$ & 0.2157 & $\mathrm{mmHg}$ \\
\hline$P_{0, p c d}$ & $\begin{array}{l}\begin{array}{l}\text { Pressure in pericardium at } 0 \\
\text { volume }\end{array} \\
\end{array}$ & 0.5003 & $\mathrm{mmHg}$ \\
\hline$P_{0, s p t}$ & $\begin{array}{l}\begin{array}{l}\text { Pressure in } \mathrm{RV} \\
\text { volume }\end{array} \\
\end{array}$ & 1.1101 & $\mathrm{mmHg}$ \\
\hline$P_{t h}$ & Pressure in the thoracic cavity & -4 & $\mathrm{mmHg}$ \\
\hline$\lambda_{\mathrm{lvf}}$ & Parameter of the EDPVR & 0.033 & $1 / \mathrm{ml}$ \\
\hline$\lambda_{\text {rvf }}$ & Parameter of the EDPVR & 0.023 & $1 / \mathrm{ml}$ \\
\hline$\lambda_{\mathrm{spt}}$ & $\begin{array}{ll}\text { Parameter of ventricular } \\
\text { interaction (VI) }\end{array}$ & 0.435 & $1 / \mathrm{ml}$ \\
\hline$\lambda_{\mathrm{pcd}}$ & Parameter of VI & 0.03 & $1 / \mathrm{ml}$ \\
\hline$V_{0, s p t}$ & Parameter of VI & 2 & $\mathrm{ml}$ \\
\hline$V_{0, p c d}$ & Parameter of VI & 200 & $\mathrm{ml}$ \\
\hline
\end{tabular}




\begin{tabular}{|llll|}
\hline \multicolumn{4}{|l|}{ Table 2 - Healthy human outputs } \\
\hline \multicolumn{2}{|l|}{ Description } & \\
\hline$V_{l v}$ & Volume in left ventricle & $111.6 / 45.6$ & $\mathrm{~mL}$ \\
\hline$S V$ & Stroke volume & 66.0 & $\mathrm{~mL}$ \\
\hline$C O$ & Cardiac output & 5.28 & $\mathrm{~L} / \mathrm{min}$ \\
\hline$P_{l v, \max }$ & Max $P_{l v}$ & 119.1 & $\mathrm{mmHg}$ \\
\hline$P_{a o}$ & Pressure in aorta & $116.5 / 79.0$ & $\mathrm{mmHg}$ \\
\hline$V_{r v}$ & Volume in the right ventricle & $112.1 / 46.1$ & $\mathrm{ml}$ \\
\hline$P_{r v, \text { max }}$ & Max $P_{r v}$ & 26.2 & $\mathrm{mmHg}$ \\
\hline$P_{p a}$ & Pressure in the pulmonary artery & $25.7 / 7.8$ & $\mathrm{mmHg}$ \\
\hline$P_{p v}$ & Pressure in the pulmonary vein & 2 & $\mathrm{mmHg}$ \\
\hline$P_{v c}$ & Pressure in the vena cava & 2 & $\mathrm{mmHg}$ \\
\hline
\end{tabular}

In Equations (1)-(9), the parameter $H(t)$ is the Heaviside function, $Q_{a v}$ and $Q_{m t}$ are the flows through the aortic valve and mitral valve of the left ventricle, $P_{a 0}, P_{p v}$ and $P_{l v}$ are the pressures in the aorta, pulmonary vein and left ventricle, $V_{l v}$ is the volume in the left ventricle, $V_{s p t}$ is the septum volume and $P_{\text {peri }}$ is the pressure in the pericardium. The Heaviside formulation of Equations (1) and (2) provides an open on pressure close on flow valve law such that:

$$
\begin{array}{ll}
L_{a v} \dot{Q}_{a v}=P_{l v}-P_{a o}-R_{a v} Q_{a v}, & Q_{m t}=0 \\
L_{m t} \dot{Q}_{m t}=P_{p v}-P_{l v}-R_{m t} Q_{m t}, & Q_{a v}=0
\end{array}
$$

where Equation (10) holds during ejection and Equation (11) during filling.

Ventricular interaction is included by modelling the septum volume $V_{\text {spt }}$ by the following equation [16]:

$$
\begin{aligned}
e(t) E_{e s, s p t}\left(V_{s p t}-V_{d, s p t}\right)+ & \left(1-e(t) P_{0, s p t}\left(e^{\lambda_{s p t}\left(V_{s p t}-V_{0, s p t}\right)}-1\right)=e(t) E_{e s, l v f t}\left(V_{l v}-V_{s p t}\right)\right. \\
& +(1-e(t)) P_{0, l v f}\left(e^{\lambda_{l v t}\left(V_{l v}-V_{s p t}\right)}-1\right)-e(t) E_{e s, r v f t}\left(V_{r v}+V_{s p t}\right) \\
- & (1-e(t)) P_{0, r v f}\left(e^{\lambda_{r v t t}\left(V_{r v}+V_{s p t}\right)}-1\right)
\end{aligned}
$$

Note that, Equation (12) is derived by setting the septum pressure volume relationship equal to the difference between the left and right ventricle pressures, for more details see [16]. Equation (12) is solved for $V_{\text {spt }}$ at each time step using a semi-analytical approach [15] for computational efficiency. 


\subsection{Simplified model}

Simulation has shown that the pressure in the pulmonary vein $P_{p v}$, and the pressure in the vena cava $P_{v c}$, typically vary in this model by only approximately $0.5 \mathrm{mmHg}$ over a cardiac cycle [26]. Thus, $P_{p v}$ and $P_{v c}$ are essentially close to constant. If $P_{p v}$ and $P_{v c}$ are held constant for the model of Figure 1, and ventricular interaction $V_{\text {spt }}$ and the pressure in the pericardium $P_{p c d}$ are set to zero, both the left and right systems of the CVS can be separated. However, note that the stroke volumes of the left and right ventricles would be the same in the measured data. Therefore, since the identification algorithm would match the left and right ventricle models to this data, there still remains an inherent coupling between the systems.

The assumptions of $V_{s p t}=0$, and $P_{p c d}=0$, are made primarily as an initial mathematical simplification to the model and to introduce modelling error to test the robustness of the derived methods. In all cases, the "measured data" used in this paper, includes both ventricular interaction and pericardium dynamics. Physiologically, the pressure in the pericardium $P_{p c d}$ is typically close to zero, but can increase significantly with pericarditis, although it still only contributes up to about $25 \%$ of left ventricular pressure $[27,28]$. Ventricular interaction can have significant effects on the right ventricle, but has less of an effect on the left ventricle [29].

Significant simulation studies have shown that changes in the inertances $L_{m t}$ and $L_{a v}$ in Figure 1 and Equations (1) and (2) do not significantly effect parameter identification [26]. The parameter $P_{0, l v f}$ has also been shown to have a limited effect [26] and for discrete data is typically identified to be close to 0 [21]. Therefore $L_{m t}, L_{a v}$ and $P_{0, l v f}$ are set to 0 . The resulting two models are shown in Figure 2, where the direction of the left ventricle-systemic system has been reversed from Figure 1 to illustrate the similarities. 


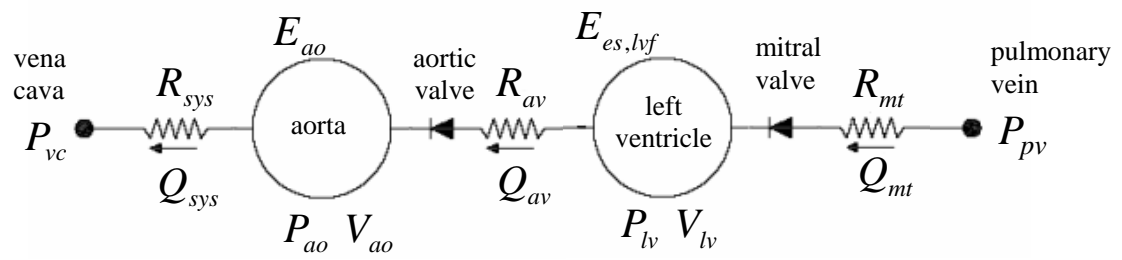

(a)

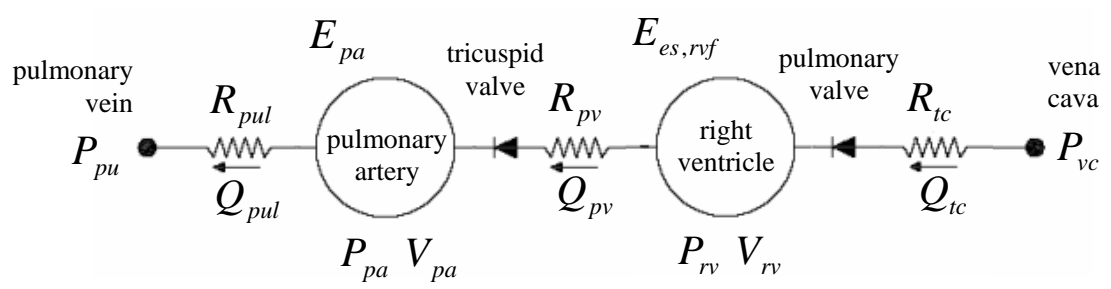

(b)

Figure 2: (a) The left ventricle-systemic system simplified model

(b) The right ventricle-pulmonary system simplified model

Replacing the input/output parameters

$$
\begin{gathered}
\text { LV Input parameters } \equiv\left\{P_{p v}, E_{e s, l v f}, E_{a o}, R_{m t}, R_{a v}, R_{s y s}, P_{v c}\right\} \\
\text { LV Output parameters } \equiv\left\{Q_{m t}, P_{l v}, V_{l v}, Q_{a v}, P_{a o}, Q_{s y s}\right\}
\end{gathered}
$$

in the left ventricle-systemic system of Figure 2(a) with the parameters :

$$
\begin{gathered}
\text { RV Input parameters } \equiv\left\{P_{v c}, E_{e s, r v f}, E_{p a}, R_{t c}, R_{p v}, R_{p u l}, P_{p u}\right\} \\
\text { RV Output parameters } \equiv\left\{Q_{t c}, P_{r v}, V_{r v}, Q_{p v}, P_{p a}, Q_{p u l}\right\}
\end{gathered}
$$

the right ventricle-pulmonary model of Figure 2(b) is obtained.

A final addition is to create an extended driver function $e(t)$ to reduce the modelling error caused by the above simplified model assumptions. The new driver function and the model differential equations for the left ventricle-systemic system of Figure 2(a) are defined:

The left ventricle-systemic system of Figure 2 (a) can be modelled: 


$$
\begin{gathered}
\dot{V}_{l v}=H\left(Q_{m t}\right) Q_{m t}-H\left(Q_{a v}\right) Q_{a v} \\
\dot{P}_{a o}=E_{a o} H\left(Q_{a v}\right) Q_{a v}-E_{a o}\left(\frac{P_{a o}-P_{v c}}{R_{s y s}}\right) \\
Q_{m t}=\frac{P_{p v}-P_{l v}}{R_{m t}} \\
Q_{a v}=\frac{P_{l v}-P_{a o}}{R_{a v}} \\
P_{l v}=e(t) E_{e s, l v f} V_{l v}+P_{t h} \\
e_{n e w}(t)=\frac{P_{l v, f u l l}-P_{t h}}{V_{l v, f u l l}}
\end{gathered}
$$

where $P_{p v}$ and $P_{v c}$ are held constant. The parameters Plv,full and Vlv,full are defined to be the full model outputs of Figure 1 from a healthy human parameter set with a heart beat period of 0.75 seconds. Hence Equation (20) represents a population driver function, which could be scaled to represent different heart beats. However, the shape could be altered as required to capture individual patients. To obtain the model equations for the Right Ventricle-pulmonary system of Figure 2(b), the parameters of Equation (13) in Equations (15)-(20) are replaced by the parameters of Equation (14).

\subsection{Healthy and disease state comparisons}

To model a diseased human, the following set of parameter changes are made from Table 1:

$$
R_{a v} \rightarrow 4 R_{a v}, R_{m t} \rightarrow 4 R_{m t}, E_{e s, l v f} \rightarrow \frac{1}{2} E_{e s, l v f}, R_{s y s} \rightarrow \frac{1}{2} R_{s y s}
$$

The changes in Equation (21) are used as an initial mathematical validation of the simplified models in Figure 2, rather than a physiologically realistic study of the clinical mechanisms involved in cardiac dysfunction. However, a halving of contractility $E_{e s, l v f}$ and systemic resistance $R_{s y s}$ is not too unrealistic for septic shock, or myocardium infarction with the addition of a vasodilator [30-33]. Furthermore, valvular stenosis can be simulated by an increase in the resistances of the aortic and 
mitral valves [30, 32, 33]. The disease states in Equation (21) would of course be unlikely to occur all at once, but it serves to provide an initial test of the methods.

\subsection{Unique Parameter identification}

The parameter identification method presented is an extension of the concept developed in [21, 26]. The idea in [21, 26] is to set up an iteration between a linear least squares optimization and a forward solution, which is partly based on a Picard iteration [34]. This approach is therefore distinctively different from other integral formulations like the modulating function approach [35] which does not iterate and is therefore not directly suitable for the discrete data and high non-linearities present in this application. Furthermore, the CVS model of Figure 1 typically requires many iterations to converge to steady state, which is highly dependent on the initial conditions. Therefore, the standard method of non-linear regression [36], is not suitable, as it is too computationally intense and can often result in local minima. In this section the method of [21] is significantly improved by avoiding the requirement of a continuous volume profile, which is typically not known. In addition the number of forward simulations and the computational requirements for each iteration are dramatically reduced.

The unknown patient specific parameters, denoted $X$, that are optimized for the left ventricle are defined:

$$
X \equiv\left\{P_{p v}, E_{e s, l v f}, E_{a o}, R_{m t}, R_{a v}, R_{s y s}\right\}
$$

The parameter $P_{v c}$ in Equation (13) is assumed known, since it would be found from either identifying the right ventricle system, or by direct measurement of the central venous pressure, which is common in an intensive care unit.

There are 6 unknown parameters in Equation (22) to be identified in the model of Figure 2 (a). Therefore, the measured maximum/minimum left ventricle volume and aortic pressure can only uniquely identify 4 of these parameters. However, the timing of the mitral valve closure corresponds to the end of the atrial contraction which can 
be detected by the end of the $\mathrm{P}$ wave on an electrocardiogram (ECG) [25]. Alternatively, since the left and right atriums contract close to simultaneously, the mitral valve closure can also be calculated from the "a wave" in the central venous pressure waveform [37]. The central venous pressure is commonly measured in the ICU.

These observations demonstrate an important concept, which is to utilize features from physiological waveforms to improve identifiability without having to explicitly model the effects. The pressure in the pulmonary vein $P_{p v}$ or the filling pressure of the simplified model of Figure 1(a) corresponds to the left ventricle pressure at the mitral valve closure. Hence, $P_{p v}$ can be estimated by the formula:

$$
P_{p v}=P_{l v}\left(t_{d 2}\right), \quad t_{d 2} \equiv \text { time of mitral valve closure }
$$

A further important feature available is the maximum gradient or inflection point in the ascending aortic pressure wave. The parameter which has a significant effect on the maximum aortic pressure gradient is the resistance in the aortic valve $R_{a v}$. Define:

$$
\alpha\left(R_{a v}\right)=\frac{P_{a o, \text { true }}\left(t_{\text {inflect }}\right)-P_{a o, \text { true }}\left(t_{\text {min }}\right)}{P_{a o, \text { approx }}\left(t_{\text {inflect }}\right)-P_{a o, \text { approx }}\left(t_{\text {min }}\right)}
$$

where $P_{a o, a p p r o x}$ and $P_{a o, t r u e}$ are the simulated and "measured" aortic pressures, $t_{\min }$ is the time of minimum aortic pressure and $t_{\text {inflect }}$ is the time of maximum aortic pressure gradient. Equation (24) is an approximation to the ratio of the maximum gradients of $P_{a o, a p p r o x}$ to $P_{a o, t r u e}$ and is used to avoid having to differentiate the aortic pressure which may be noisy. Simulation has shown that the variable $\alpha$ in Equation (24), changes inversely proportional to $R_{a v}$ with all other parameters held at their nominal values. Specifically, if $R_{a v}$ increases by a factor of 2, with all other parameters fixed, $\alpha$ approximately reduces by a factor of 2 , with a order of magnitude less effect on the maximum and minimum volumes/pressures. This result motivates an approximation to $R_{a v}$ : 


$$
R_{a v, \text { approx }}=\frac{P_{a o, a p p r o x}\left(t_{\text {inflect }}\right)-P_{a o, a p p r o x}\left(t_{\min }\right)}{P_{a o, \text { true }}\left(t_{\text {inflect }}\right)-P_{a o, \text { true }}\left(t_{\min }\right)}
$$

However, for Equation (23) and (25) to be valid approximations, to $P_{p v}$ and $R_{a v}$, the approximations $P_{l v, a p p r o x}$ and $P_{a o, a p p r o x}$ need to be as accurate as possible. The solution proposed, is to first ensure that the maximum/minimum simulated volumes and aortic pressures are precisely matched to the measured values for given initial (but essentially arbitrary) estimates of $P_{p v}$ and $R_{a v}$. At the end of this optimization, $P_{p v}$ and $R_{a v}$ are updated using Equations (23) and (25).

Simulation has shown that increasing the parameters $E_{e s, l v f}$, and $R_{m t}$ separately by factors of 2 decrease the mean volume, and stroke volume by factors close to 2 . On the other hand, increasing the parameters for $E_{a o}$ and $R_{s y s}$ proportionally increase the pulse pressure difference and the mean aortic pressure. These results motivate the following definitions:

$$
\begin{aligned}
E_{e s, l v f, \text { approx }} & =\left(\frac{V_{l v, \text { min,approx }}+V_{l v, \text { max }, \text { approx }}}{V_{l v, \text { min,true }}+V_{l v, \text { max }, \text { true }}}\right) E_{\text {es }, \text { lvf }, \text { old }} \\
R_{\text {mt,approx }} & =\left(\frac{S V_{\text {approx }}}{S V_{\text {true }}}\right) R_{m t, \text { old }} \\
E_{\text {ao,approx }} & =\left(\frac{P P_{\text {true }}}{P P_{\text {approx }}}\right) E_{a o, o l d} \\
R_{\text {sys,approx }} & =\left(\frac{P_{a o, \text { max }, \text { true }}+P_{a o, \text { min,true }}}{P_{a o, \text { max }, \text { approx }}+P_{a o, \text { min }, \text { approx }}}\right) R_{\text {sys }, \text { old }}
\end{aligned}
$$

Consider $R_{m t, a p p r o x}$ in Equation (27). Integrating Equation (17) over one heart beat yields:

$$
R_{\text {mt,true }}=\frac{\int_{0}^{\text {period }}\left(P_{p v}-P_{l v, \text { true }}\right)}{S V_{\text {true }}}
$$

For a given $P_{p v}$, let $\bar{R}_{m t}$ be the current estimate of $R_{m t, \text { true }}$, with corresponding approximations $\bar{P}_{l v}$ and $\overline{S V}$ to $P_{l v, \text { true }}$ and $S V_{\text {true }}$. Therefore: 


$$
\bar{R}_{m t}=\frac{\int_{0}^{p e r i o d}\left(P_{p v}-\bar{P}_{l v}\right)}{\overline{S V}}
$$

Dividing Equation (30) by Equation (31) yields:

$$
\frac{R_{m t, \text { true }}}{\bar{R}_{m t}}=\frac{\overline{S V}}{S V_{\text {true }}} \times \frac{\int_{0}^{\text {period }}\left(P_{p v}-P_{l v, \text { true }}\right)}{\int_{0}^{\text {period }}\left(P_{p u}-\bar{P}_{l v}\right)}
$$

Assuming that $\bar{P}_{l v}$ is much closer to $P_{l v, \text { true }}$ than $\overline{S V}$ is to $S V_{\text {true }}$ it follows that:

$$
R_{m t, \text { true }} \square \bar{R}_{m t} \times \frac{\overline{S V}}{S V_{\text {true }}}
$$

Therefore, Equation (27) also follows from an integral formulation of Equation (17) over one heart beat period. A similar approach (not shown) can be used to derive Equations (26), (28) and (29).

Note that an alternative approach would be to scale the approximate waveform $\bar{V}_{l v}$ so that $\overline{S V}=S V_{\text {true }}$, then evaluate the ratio of the integrals in Equation (32). However, evaluating Equation (32) directly, which is effectively the method of [21], relies on approximating the continuous left ventricle waveform $V_{l v}$ throughout the heart beat, which can introduce errors. In prior work [21], the estimates and the accuracy of convergence often relied on reasonable starting waveform shapes, and in some cases did not converge satisfactorily without some manual intervention. The method of Equations (26)-(29) only requires discrete data and is similar to proportional feedback control. Specifically, the parameters in Equations (26)-(29) continually change until the ratios are driven to one and is thus fully automatic.

The method presented is also readily generalizable to parameter identification of other models, by locating the major geometric effects of each parameter on the measured data, and formulating a control system like Equations (26)-(29), to iterate the parameters. The specific equations that enable the parameters to converge are of 
course model specific, but the overall approach and concept, including the breaking down of complex models into sub-models that are fully identifiable, is general.

The tests for the parameter identification method are done first in simulation with noise, and then on animal data. In the tests with noise, the "measured data" is taken to be the output of the six chamber model of Figure 1. For the animal experiments, the measured data is from catheters [38]. In both cases:

$$
\text { measured data } \equiv \text { mean } V_{l v}, S V, P P \text {, mean } P_{a o}, P_{a o}(t), t_{d 2}
$$

where:

$$
\begin{aligned}
& \text { mean } V_{l v}=\frac{V_{l v, \text { max }}+V_{l v, \text { min }}}{2}, S V=V_{l v, \text { max }}-V_{l v, \text { min }}, P P=P_{a o, \text { max }}-P_{a o, \text { min }} \\
& \text { mean } P_{a o}=\frac{P_{a o, \text { max }}+P_{a o, \text { min }}}{2}, t_{d 2} \equiv \text { end diastolic time }
\end{aligned}
$$

The overall parameter identification method is summarized in Figure 3.

$\underline{\text { Step } 1}$ Choose arbitrary set of input parameters including $P_{p v}$ and $R_{a v}$

Step 2 Simulate model of Equations (15)-(20)

Step 3 Compute approximations to $E_{e s, l v f}, R_{m t}, E_{a o}$ and $R_{s y s}$ from Equations (25)-(28).

Step 4 Simulate model of Equations (15)-(20).

$\underline{\text { Step } 5}$ If the maximum volumes and aortic pressures are matched within a given tolerance to data in Equation (34), go to Step 6, otherwise go back to Step 3.

Step 6 Compute $P_{p v}$ and $R_{a v}$ from Equations (23) and (25). If they have changed by less than $1 \%$ go to Step 7 otherwise go back to Step 3.

$\underline{\text { Step } 7}$ Output final solution and identified parameters

Figure 3: Parameter identification algorithm for Figure 2(a) 


\subsection{No volume measurement}

The algorithm of Figure 3 can be readily extended to the case where the mean $V_{l v}$ in Equation (34) is removed. The method is to let the mean $V_{l v}$ be an extra unknown parameter and optimized along with the parameters in Equation (22). To account for the increase in unknown parameters, more information from the aortic pressure waveform is used. Define:

$$
\begin{gathered}
\operatorname{error}\left(\text { mean } V_{l v}\right)=\sqrt{\left.\sum_{i=1}^{n}\left(P_{\text {ao,approx }}\left(t_{i}\right)-P_{\text {ao,true }}\left(t_{i}\right)\right)^{2}\right)} \\
t_{1}=t_{\text {min }}, t_{n}=t_{\text {min }}+3\left(t_{\text {inflect }}-t_{\text {min }}\right) \\
n \equiv \# \text { equally spaced time points } \in\left[t_{1}, t_{n}\right]
\end{gathered}
$$

where $t_{\min }$ and $t_{\text {inflect }}$ are from Equation (25), and $n$ is on the sampling frequency of the aortic pressure catheter which is taken to be $1 \mathrm{kHz}$. The metric error is dependent on the mean $V_{l v}$ and the points $t_{1}$ and $t_{n}$ are equally spaced about $t_{\text {inflect }}$. It is important to note that the full aortic pressure waveform cannot be used in Equation (36). The reason is that the model does not capture the dicrotic notch so matching to this part of the waveform would introduce unnecessary error into the method. However, the continuous waveform in the interval $\left[t_{1}, t_{n}\right]$ still provides significantly extra data that can be used to identify the mean volume and thus the maximum/minimum left ventricle volumes as well.

The method starts with an approximation $V_{l v, \text { max,approx }}$ to the maximum volume. The approximate mean volume is thus defined:

$$
\text { mean } V_{l v, \text { approx }}=V_{l v, \text { max }, \text { approx }}-\frac{S V}{2}
$$

The algorithm of Figure 3 is then applied and the error in Equation (36) is computed. The maximum volume $V_{l v, \text { approx,max }}$ is then updated in a depth first search to minimize the error of Equation (36). The method is summarized in Figure 4. 
Step 1 Choose a value of $V_{l v, a p p r o x, \max }$, and determine the mean $V_{l v}$ from Equation (38).

$\underline{\text { Step } 2}$ Apply the method of Figure 3 to identify $P_{p v}, R_{a v}, E_{e s, l v f}, R_{m t}, E_{a o}$ and $R_{\text {sys. }}$

$\underline{\text { Step } 3}$ Compute the error of Equation (36).

$\underline{\text { Step } 4}$ Repeat Steps 1-3 in a depth first search until $V_{l v, a p p r o x, \max }$ changes by less than $1 \%$.

Step 5 Output the final approximation $V_{l v, a p p r o x, \max }$ and identified parameters

Figure 4: Parameter identification algorithm for Figure 2(a) without a volume measurement, but a known stroke volume.

\section{Results and Discussion}

This section first validates the simplified modelling approach of Figures 2(a) and (b), and the parameters identification method of Figure 3, against simulated data from the full model of Figure 1. Measurement noise is simulated by corrupting the simulated data with random uniformly distributed noise. Due to the symmetry of Figures 2(a) and (b) only tests on the left-ventricle system are considered. The noise is defined:

$$
\text { uniform noise } \equiv 5 \% \text { in } P_{a o}(t), 10 \% \text { in SV, } 10 \% \text { in } t_{d 2}
$$

where for the end-diastolic filling time $t_{d 2}$ in Equation (34), the $10 \%$ noise is relative to the length of time of the diastole. The noise is made less for the pressure, since it is assumed that a catheter measures the aortic pressure waveform, which is standard in an ICU. Modelling error is also present in the simplified models of Figure 2 with respect to the full model of Figure 1. 
The model and methods of the left ventricle-systemic system are then tested on clinical data from a pulmonary embolism animal model experiment [39].

\subsection{Convergence of algorithm and effectiveness of modelling approach}

To demonstrate the fast and accurate convergence of the algorithm of Figure 3, and to assess the suitability of the simplified models of Figure 2 in describing the full model of Figure 1, a healthy human is first considered. The parameters for a healthy human are given in Tables 1-2. In this case no noise is added so an accurate characterization of the accuracy of the simplified models can be obtained. The assumed measured data is:

$$
\text { measured data } \equiv \text { mean } V_{l v}, S V, P P \text {, mean } P_{a o}, P_{a o}(t), t_{d 2}
$$

where $P_{a o}$ is given as a function of time since it is continuously measured.

The left ventricle volume matches very closely to the true volume with maximum errors of $1.6 \%$ and $2.6 \%$ during filling and ejection, and errors of $0.00016 \%$ and $0.00014 \%$ in the maximum and minimum volumes respectively. Similarly, the identified aortic pressure closely captures the true pressure with maximum errors of $1.8 \%$ and $0.2 \%$ during ejection and filling, and errors of $0.0026 \%$ and $0.0015 \%$ in the maximum and minimum aortic pressures respectively. The error in the maximum ventricle pressure is $2.1 \%$.

For the identified parameters, the largest error is $21.7 \%$ in $R_{a v}$ but this is largely due to the already small value. There is also an error of $8.1 \%$ in $E_{e s, l v f}$ which reflects the modelling error of Figure 2 with respect to Figure 1. These results show that the simplified model of Equations (15)-(20) is a very accurate representation of the full model of Figure 1, with small errors due to modelling error. 
The accurate results also provide an initial validation of the parameter identification method of Figure 3, with very fast convergence obtained, even when starting significantly far away from the solution. Figure 5 plots the maximum volume and Figure 6 plots $R_{a v}$ against the number of iterations, for an initial guess containing 300$400 \%$ error in all the parameters. One iteration is equivalent to one numerical simulation of Equations (15)-(20) that occurs in step 3 of Figure 3. The maximum volume converges in about 10 iterations, and remains largely unaffected by changes in $R_{a v}$, where $R_{a v}$ takes about 24 iterations to converge within $1 \%$. The convergence time of $R_{a v}$ can be reduced by a factor of 2 by re-defining $R_{a v}$ in Equation (25) by:

$$
R_{a v, \text { approx }}=\left(\frac{P_{a o, \text { approx }}\left(t_{\text {inflect }}\right)-P_{a o, \text { approx }}\left(t_{\min }\right)}{P_{a o, \text { true }}\left(t_{\text {inflect }}\right)-P_{a o, \text { true }}\left(t_{\min }\right)}\right)^{\text {gain }} R_{a v, \text { old }}
$$

and setting a gain of 3, as shown in Figure 6. Importantly, once the method converges, this implies that the ratios in Equations (26)-(29) must be unity, otherwise the parameters would keep changing. Hence, the method can never reach a local minima and must always stop at the global minimum. 


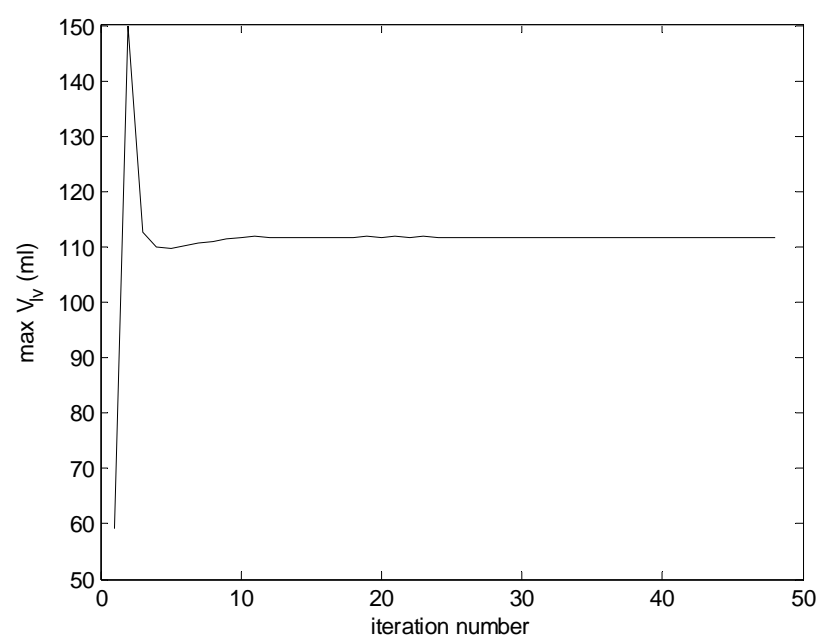

Figure 5: Convergence of the maximum volume using algorithm of Figure 3.

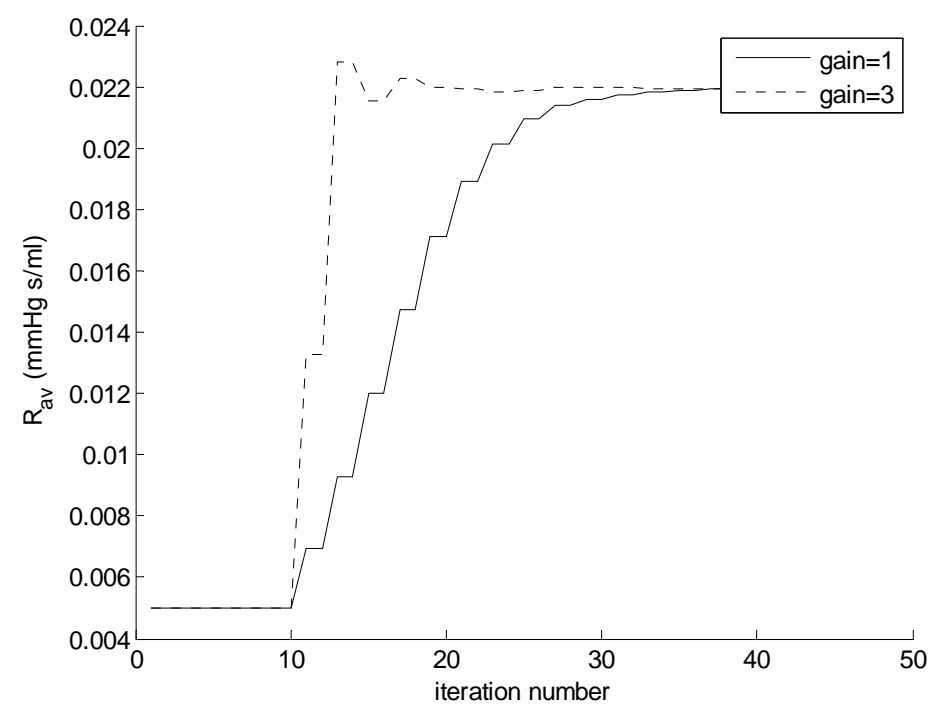

Figure 6: Convergence of $R_{a v}$ in the algorithm of Figure 3 using two gains of 1 and 3 in Equation (41).

Similarly accurate results are obtained for the diseased state human of Equation (21), so these results are not shown. In summary the parameter identification method of Figure 3 is very fast and accurate independent of starting point, and the simplified models of Figure 2 closely capture the full model dynamics of Figure 1. Importantly, the data of Equation (34) is sufficient to uniquely identify all six parameters in 
Equation (22). The simpler nature of Figure 2 also means the model simulations are dramatically faster.

\subsection{Healthy and diseased human with noise - no volume measurement}

100 Monte Carlo simulations are performed for the algorithm of Figure 4 with noise levels defined in Equation (39). To optimize accuracy, a cubic smoothing spline is performed on the noisy aortic pressure waveform using standard in-built functions in Matlab. The identified parameters are summarized in Table 3:

\begin{tabular}{|l|l|l|l|l|}
\hline Parameters & \multicolumn{3}{|l|}{ Healthy } & Diseased \\
\hline & Median & $90 \% \mathrm{CI}$ & Median & $90 \% \mathrm{CI}$ \\
\hline $\mathrm{P}_{\mathrm{pv}}$ & 1.7 & {$[0.8,3.0]$} & 3.7 & {$[1.2,7]$} \\
\hline $\mathrm{R}_{\mathrm{av}}$ & 0.012 & {$[0.006,0.02]$} & 0.064 & {$[0.038,0.082]$} \\
\hline $\mathrm{E}_{\mathrm{es}, \mathrm{lvf}}$ & 2.3 & {$[2.0,2.8]$} & 1.1 & {$[0.9,1.3]$} \\
\hline $\mathrm{R}_{\mathrm{mt}}$ & 0.015 & {$[0.01,0.02]$} & 0.061 & {$[0.042,0.09]$} \\
\hline $\mathrm{R}_{\text {sys }}$ & 1.10 & {$[1.00,1.20]$} & 0.53 & {$[0.49,0.58]$} \\
\hline $\mathrm{E}_{\mathrm{ao}}$ & 0.70 & {$[0.64,0.75]$} & 0.68 & {$[0.62,0.75]$} \\
\hline
\end{tabular}

Table 3: Comparing the identified parameters for a healthy and diseased human with noise from Equation (39).

The median ratios of $E_{e s, l v f, d i s e a s e} / E_{e s, l v f, h e a l t h y}, R_{a v, \text { disease }} / R_{a v, \text { healthy, }}, R_{m t, \text { disease }} / R_{m t \text {,healthy, }}$ and $R_{\text {sys,disease }} / R_{\text {sys,healthy }}$ are $0.48,5.3,4.1$ and 0.48 respectively, which capture the reduced contractility, increased resistances and reduced systemic resistances as defined in Equation (20). There are also good separations in the 90\% CI's. In addition, 90\% of the identified maximum volumes have an error less than $11.5 \%$ and $14.7 \%$ for the diseased and healthy human respectively. However, part of this error is due to the overestimation of the volume in each case. Taking the identified volumes with no noise as the "true" volumes gives $90 \%$ of all errors less than $8.7 \%$ and $12.2 \%$ 
respectively. Furthermore, 90\% of the identified maximum left ventricle pressures have an error less than $1.5 \%$ and $9.4 \%$, for the healthy and disease states.

\subsection{Validation on an animal model and clinical implementation}

To demonstrate the clinical potential for the methods developed, data from a porcine pulmonary embolism experiment is used. The data is obtained from the Hemodynamics Research Laboratory, University of Liege, Belgium. In the experiments, a pig is injected with autologous blood clots every two hours to simulate pulmonary embolism [38].

As a simple proof of concept, the left ventricle model of Figure 1(a) and the method of Figure 2 are applied using measured waveforms for one of the pigs at two time points of 30 minutes and 210 minutes. No ECG or the central venous pressure waveform was available, therefore, the end-diastolic filling time $t_{d 2}$ in Equation (34) was manually estimated from the left ventricle volume profile. A driver function is derived in a similar way to Equation (20), but with $P_{l v, f u l l}$ and $V_{l v, f u l l}$ replaced by the measured left ventricle pressure and volume, and $P_{t h}$ is set to 0 , since the pig is open chest. The resulting function is smoothed by least squares cubic splines and normalized so the maximum point is 1 and the time interval of one heart beat is the healthy value of 0.75 . To account for different heart rates, the generic shape is defined:

$$
\hat{e}(t)=\bar{e}\left(\frac{0.75}{\text { period }} t\right)
$$

where $\bar{e}(t)$ is experimentally derived from the healthy state of several pigs based on an average response and is shown in Figure 7. 


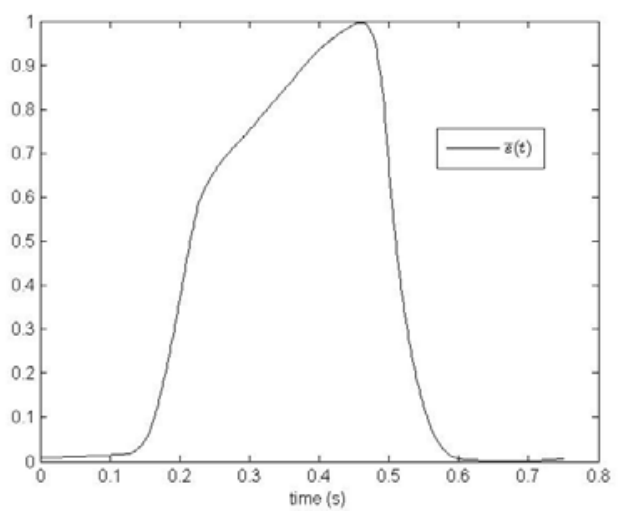

Figure 7: Experimentally derived driver function $\bar{e}(t)$ based on Equation (20)

To account for individual pig variations the final driver function is defined:

$$
\begin{aligned}
e(t) & =\hat{e}(\alpha t+\beta), \quad 0<t<\text { period } \\
\alpha & =\frac{t_{\nabla a o, \min }-t_{a o, \min }}{t_{\text {inflect }, 2}-t_{\text {inflect }, 1}}, \beta=\frac{t_{a o, \text { min }} t_{\text {inflect }, 2}-t_{\nabla a o, \text { min }} t_{\text {inflect }, 1}}{t_{\text {inflect }, 2}-t_{\text {inflect }, 1}}
\end{aligned}
$$

where $t_{a o, \min }$ is the time of minimum aortic pressure, $t_{\nabla a o, \min }$ is the time of the minimum (or maximum negative) aortic pressure gradient, and $t_{\text {inflect }, 1}$ and $t_{\text {inflect }, 2}$ are the first and second inflection points of $\hat{e}$ in Equation (43). Specifically, $t_{\nabla a o, \min }$ is well known to correspond to the minimum left ventricle pressure gradient (or inflection point) which always occurs just before the dicrotic notch, and thus corresponds to the aortic valve closure. The volume is approximately constant at this point, and therefore, the formula of Equation (20) shows that $t_{\text {inflect, } 2}$ should be equal to $t_{\nabla a a, \min }$. The maximum left ventricle pressure gradient, is also known to occur just before the aortic valve opens, which corresponds closely to $t_{a o, \min }$. Therefore since the volume is constant at this point as well, $t_{\text {inflect }, 1}$ should be equal to $t_{a o, \min }$. The time scaling transformation in Equation (43) ensures that the inflection points of the driver function correspond to $t_{a o, \min }$ and $t_{\nabla a o, \min }$ as required. Equations (42) and (43) therefore provide a way of approximately identifying a patient (pig) specific driver function. Further clinical experiments and trials on humans are needed to classify to what degree of accuracy the driver function is required to be for adequate cardiac diagnosis. 


\subsubsection{Parameter identification with known volumes}

Figure 8(a) shows the result of applying the algorithm of Figure 3 on the one of the pigs at 30 minutes into the pulmonary embolism experiment. This figure is compared to a special case of a fixed $P_{p v}=2$ and $R_{a v}=0.46$ in Figure 8(b). In both cases the maximum and minimum values of $V_{l v}$ and $P_{a o}$ (not shown) are accurately captured, but there are errors of $34 \%$ and $84 \%$ in the parameters $E_{e s, l v f}$ and $R_{m t}$. The errors in $E_{a o}$ and $R_{\text {sys }}$ are less than 5\%. However, the parameter $E_{\text {es,lvf }}$ appears relatively robust and is virtually unaffected by changes in $P_{p u}$. For example if $R_{a v}<0.2$, the errors of $E_{e s, l v f}$ are less than $10 \%$. But these results highlight the importance of the data set in Equation (40) to accurately identify the model as well as finding a unique parameter set.

The PV curve and aortic pressure waveform corresponding to the correct parameters in Figure 8(a) are plotted in Figure 9, showing a close match. Notice how the first third to a half of the ascending aortic pressure is matched almost exactly. The high degree of accuracy in this period is due to the parameter identification method forcing the inflection point of the model to match the inflection point of the data. This result further shows the power of the method of Figure 3 as any feature that the model of Figure 2(a) is capable of matching, can be precisely captured independent of starting point, with very fast convergence and very minimal computation. 
(a)

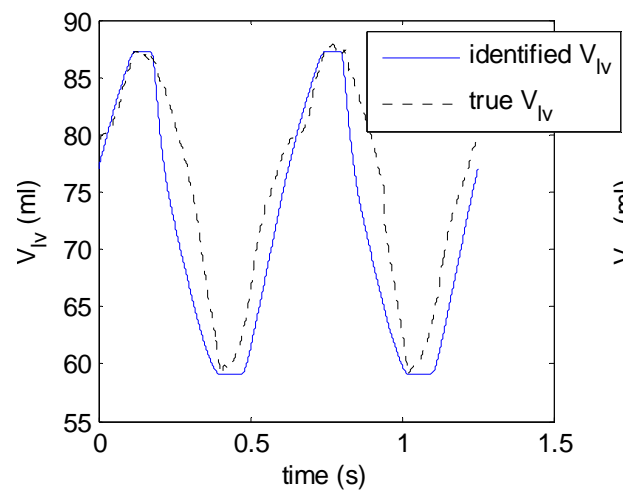

(b)

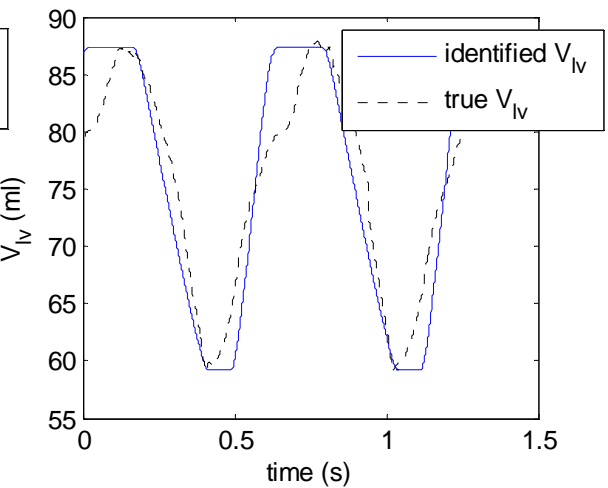

Figure 8: Applying the algorithm of Figure 3 to the pig data. (a) identifying all parameters (b) fixing $P_{p v}$ and $R_{a v}$

(a)

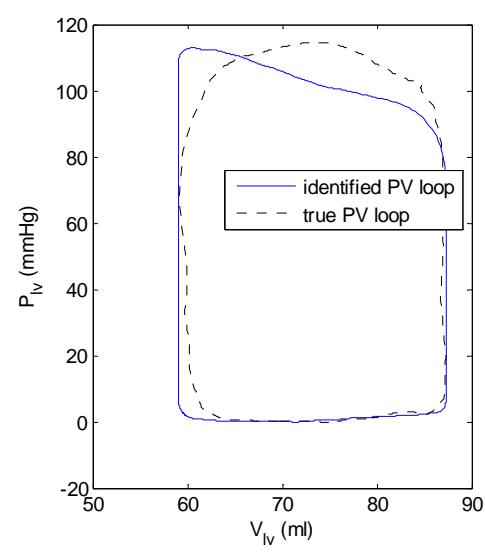

(b)

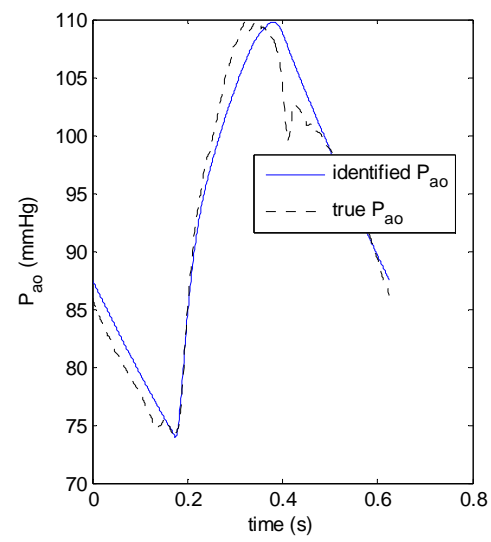

Figure 9: Identification results using correct parameters of Figure 8(a). (a) Pressurevolume curve (b) Aortic pressure waveform

It has been shown that the ascending aortic waveform inflection point is a predictive factor for all-cause and cardiovascular mortality in patients with chronic renal failure on hemodialysis [40]. Many other studies have also shown features in the continuous aortic pressure waveform to help diagnose disease states and to monitor improvements due to therapy. Therefore, this modelling and parameter identification 
approach has the potential to aggregate key clinical information and any significant correlations between parameters observed in the literature.

\subsubsection{Parameter identification without volumes}

The method of Figure 4 with mean $V_{l v}$ as an extra unknown, is applied on two separate time periods, at 30 minutes and 120 minutes. The results of the identified parameters are compared to the parameters identified with the algorithm of Figure 3, which are treated as the "true" parameters.

For the pig at 30 minutes, the method identified $R_{m t}, R_{s y s}, E_{a o}$ and $P_{p v}$ with an accuracy less than $3 \%$ of the true values, with errors of $7.6 \%$ in $E_{e s, l v f}$ and $18.8 \%$ in $R_{a v}$. However the larger error in $R_{a v}$ can be attributed to the small size of the true value for $R_{a v}$. The errors for the identified left ventricle volume and pressure were $4.6 \%$ and $1.0 \%$. For the pig at 120 minutes, the method identified $R_{m t}, R_{s y s}, E_{a o}$ and $P_{p v}$ with an accuracy less than $2 \%$ of the true values, with errors of $5.1 \%$ in $E_{e s, l v f}$ and $14.7 \%$ in $R_{a v}$. These results combined with the simulated results in the prior section suggest that the volume may not be needed to identify parameters, but requires further validation in clinical trials. These trials would need to use either ECG or the central pressure waveform to calculate the end diastolic filling time. In addition, it is required to

determine/define what errors in $V_{l v \text {, max }}$ are acceptable for diagnosis. In summary, the simulated data and the pig data with a manually chosen end diastolic filling time from the volume profile, prove the concept that the reduced data set of Equation (70) is potentially sufficient for diagnosis and warrants further investigation.

\section{Summary and relevance to Biomedical Engineering}

Three major concepts were introduced in this paper.

- Simplified fully identifiable sub-models that closely capture a six chamber model with ventricular interaction, pericardium and inertial effects, and are capable of uniquely characterizing measured clinical response of pig data 
- A parameter identification method based on a proportional feedback control system that iterates between a forward solution and parameter updates until the measured clinical data is precisely matched. The formulation also allows any geometrical feature that the model is capable of producing, to be precisely captured with very minimal computation, for example the end-diastolic filling time and maximum ascending aortic gradient. Once the method converges, by definition of the control system reference input, the global minimum must be reached. Therefore there can be no local minima which can commonly occur in the more traditional non-linear regression.

- The reduction of the measured data set by including the removed measurements as extra unknowns in the identification method. A fast and accurate parameter identification makes this reduction possible, with minimal effect on computation. Results in both simulation and clinical data suggest the maximum and minimum volumes are not needed for disease state diagnosis, with the addition of end-diastolic filling time and continuous ascending aortic pressure.

Current models e.g. [41, 42] are good at capturing trends in data but do not typically capture precise quantitative pressure and volume changes, including the exact measured valve timing and ascending aortic pressure profile, for individual patients. Of course in principal, complex models could be made to match to any given data set. But for limited data in an ICU setting, unique parameter identification would only be obtained if a small subset of the parameter set is optimized. Thus, the majority of parameters have to be fixed at generic values which are only ever known on a population level, not for individual patients [43-45]. Therefore, pre-determined dynamics are assumed, which are likely wrong in fast changing critical care patients. Furthermore, a relatively large amount of computation is spent simulating these dynamics from the fixed parameters, which has no direct effect on matching a patient.

The approach in this paper is different as it only simulates the precise subset of the model that is being identified to the data. Therefore, very fast and unique parameter identification methods can be developed that can capture virtually any desired features with minimal effect on computational time, high accuracy and avoidance of 
local minima's. For example, the methods in this paper quantitatively capture the measured valve timing, where usually the qualitative trend is reported [42]. In addition, a patient specific driver function is approximately identified, without requiring the measured pressure volume curve [46].

Finally, the completely patient specific approach in this paper, and the fast identification methods gives the potential to analyze clinical data in an ICU of time periods of days or weeks with minimal computational time. The identified parameter changes can then be characterized as time progresses. The result would be the creation of a more complex patient specific models in real-time, without any preassumptions on patient behaviour, for example reflex response [41, 45]. This paper therefore represents a set of modelling and computational tools with the potential to monitor and better manage the cardiovascular system in critical care patients.

\section{Conclusions}

Two simplified models of the left ventricle systemic and right ventricle pulmonary systems were developed that closely matched output data from a full cardiovascular system model with pericardium and ventricular interaction dynamics. The left ventricle system model was tested in simulation with up to $10 \%$ random uniformly distributed noise added to the data. The model was shown to be uniquely identifiable with the addition of the end-diastolic filling time and continuous information from the aortic pressure waveform. Furthermore, the extra data used, that is readily available in an ICU, enabled the mean volume to be added as an extra unknown parameter with minimal effect on identifiability. This result has significant potential clinically, as the mean or maximum/minimum volumes are much harder to measure, where the stroke volume is relatively easy and more common.

The approach of breaking down the six chamber heart model into separate uniquely identifiable models is general and could be applied to any complex lumped parameter CVS model. In particular, future work will utilize the simpler models to allow rapid identification of the 8 chamber model [22] and any other added dynamics as required to diagnose cardiac disease states and characterize therapy response. 
The clinical and simulated results both suggest that potentially better model-based diagnostic capability could be obtained with the addition of continuous aortic pressure information, and either ECG or the central venous pressure waveform to obtain the end-diastolic time. This enhanced capability has been shown to be not significantly reduced when removing volume $V_{l v, \text { max }} / V_{l v, \text { min }}$ measurements. The results thus show the potential for practical implementation of a model-based cardiac diagnosis/therapeutics system in the ICU based on readily measurable parameters.

\section{References}

[1] Franklin C. and J. Mathew, "Developing strategies to prevent inhospital cardiac arrest: analyzing responses of physicians and nurses in the hours before the event," Critical care medicine, vol. 22, pp. 244-7, Feb 1994.

[2] Perkins G. D., D. F. McAuley, S. Davies, and F. Gao, "Discrepancies between clinical and postmortem diagnoses in critically ill patients: an observational study," Critical care (London, England), vol. 7, pp. R129-32, Dec 2003.

[3] Smith W. R., R. M. Poses, D. K. McClish, E. C. Huber, F. L. Clemo, D. Alexander, and B. P. Schmitt, "Prognostic judgments and triage decisions for patients with acute congestive heart failure," Chest, vol. 121, pp. 1610-7, May 2002.

[4] Angus D. C., W. T. Linde-Zwirble, J. Lidicker, G. Clermont, J. Carcillo, and M. R. Pinsky, "Epidemiology of severe sepsis in the United States: analysis of incidence, outcome, and associated costs of care," Critical care medicine, vol. 29, pp. 1303-10, Jul 2001.

[5] Brun-Buisson C., "The epidemiology of the systemic inflammatory response," Intensive care medicine, vol. 26 Suppl 1, pp. S64-74, 2000.

[6] D. C. Angus, M. A. Kelley, R. J. Schmitz, A. White, and J. Popovich, Jr., "Caring for the critically ill patient. Current and projected workforce requirements for care of the critically ill and patients with pulmonary disease: can we meet the requirements of an aging population?," Jama, vol. 284, pp. 2762-70, Dec 62000.

[7] Ewart G. W., L. Marcus, M. M. Gaba, R. H. Bradner, J. L. Medina, and E. B. Chandler, "The critical care medicine crisis: a call for federal action: a white paper from the critical care professional societies," Chest, vol. 125, pp. 151821, Apr 2004.

[8] Kelley M. A., D. Angus, D. B. Chalfin, E. D. Crandall, D. Ingbar, W. Johanson, J. Medina, C. N. Sessler, and J. S. Vender, "The critical care crisis in the United States: a report from the profession," Chest, vol. 125, pp. 1514-7, Apr 2004. 
[9] Kerckhoffs R. C. P., M. L. Neal, Q. Gu, J. B. Bassingthwaighte, J. H. Omens, and A. D. McCulloch, "Coupling of a 3D finite elment model of cardiac ventricular mechanics to lumped systems of the systematic and pulmonic circulation.," Biomed. Eng., vol. 35, pp. 1-18, 2007.

[10] Hunter P. J. and B. H. Smaill, Structure and function of the diastolic heart: material properties of passive myocardium, in: L.Glass,P.Hunter,A.McCulloch (Eds.), Theory of Heart: Springer-Verlag, Harrisonburg, 1991.

[11] Legrice I. J., P. J. Hunter, and B. H. Smaill, "Laminar structure of the heart: mathematical model," Am.J.Physiol., vol. 272, p. H2466-H2476, 1997.

[12] Chung D. C., S. C. Niranjan, J. W. Clark Jr, A. Bidani, W. E. Johnston, J. B. Zwischenberger, and D. L. Traber, "A dynamic model of ventricular interaction and pericardial influence," Am. J. Physiol., vol. 272, pp. H29422962, 1997.

[13] Luo C., D.L. Ware, J.B. Zwischenberger, and J. W. C. Jr, "Using a human cardiopulmonary model to study and predict normal and diseased ventricular mechanics, septal interaction, and atrio-ventricular blood flow patterns," $J$. Cardiovasc. Eng., vol. 7, pp. 17-31, 2007.

[14] Mukkamala R. and R. J. Cohen, "A forward model-based validation of cardiovascular system identification," Am. J. Physiol. Heart Circ. Physiol., vol. 281, pp. H2714-H2730, 2001.

[15] Hann C. E., J. G. Chase, and G. M. Shaw, "Efficient implementation of nonlinear valve law and ventricular interaction dynamics in the minimal cardiac model," Comput Methods Programs Biomed, vol. 80(1), pp. 65--74, Oct 2005.

[16] Smith B. W., J. G. Chase, R. I. Nokes, G. M. Shaw, and G. Wake, "Minimal haemodynamic system model including ventricular interaction and valve dynamics," Medical Engineering \& Physics, vol. 26(2), pp. 131-139, 2004.

[17] Pillonetto G., G. Sparacino, and C. Cobelli, "Numerical non-identifiability regions of the minimal model of glucose kinetics: superiority of Bayesian estimation," Math Biosci, vol. 184, pp. 53-67, 2003.

[18] Thomaseth K. and C. Cobelli, "Generalized sensitivity functions in physiological system identification.," Ann. Biomed. Eng., vol. 27, pp. 607-616, 1999.

[19] Audoly S., L. D'Angio, M. P. Saccomani, and C. Cobelli, "Global identifiability of linear compartmental models--a computer algebra algorithm," IEEE Trans Biomed Eng, vol. 45, pp. 36-47, Jan 1998.

[20] Audoly S., G. Bellu, L. D'Angio, M. P. Saccomani, and C. Cobelli, "Global identifiability of nonlinear models of biological systems," IEEE Trans Biomed Eng, vol. 48, pp. 55-65, Jan 2001.

[21] Starfinger C., C. E. Hann, J. G. Chase, T. Desaive, A. Ghuysen, and G. M. Shaw, "Model-based cardiac diagnosis of pulmonary embolism," Comput Methods Programs Biomed, vol. 87(1), pp. 46--60, Jul 2007.

[22] Starfinger C., J. G. Chase, C. E. Hann, G. M. Shaw, B. Lambermont, A. Ghuysen, P. Kolh, P. Dauby, and T. Desaive, "Model-based identification and diagnosis of a porcine model of induced endotoxic shock with hemofiltration," Math Biosci, vol. 216(2), pp. 132-139, 2008.

[23] Starfinger C., J. G. Chase, C. E. Hann, G. M. Shaw, P. Lambert, B. W. Smith, E. Sloth, A. Larsson, S. Andreassen, and S. Rees, "Model-based identification of PEEP titrations during different volemic levels," Computer Methods and Programs in Biomedicine, vol. 91(2), pp. 135-144, 2008. 
[24] Starfinger C., J. G. Chase, C. E. Hann, G. M. Shaw, P. Lambert, B. W. Smith, E. Sloth, A. Larsson, S. Andreassen, and S. Rees, "Prediction of hemodynamic changes towards PEEP titrations at different volemic levels using a minimal cardiovascular model," Computer Methods and Programs in Biomedicine, vol. 91(2), pp. 128-134, 2008.

[25] Guyton A. C. and J. E. Hall, Textbook of medical physiology, 10th ed.: W.B. Saunders Company, Philadelphia, 2000.

[26] Hann C. E., J. G. Chase, and G. M. Shaw, "Integral-based identification of patient specific parameters for a minimal cardiac model," Comput Methods Programs Biomed, vol. 81(2), pp. 181--192, Feb 2006.

[27] Shabetai R., The Pericardium: Kluwer Academic Publishers, 2003.

[28] Sutton J. and D. G. Gibson, "Measurement of postoperative pericardial pressure in man," British Heart Journal, vol. 39, pp. 1-6, 1977.

[29] Santamore W. P. and D. Burkhoff, "Hemodynamic consequences of ventricular interaction as assessed by model analysis," American Journal of Physiology, vol. 260, pp. H146-H157, 1991.

[30] Smith B. W., S. Andreassen, G. M. Shaw, P. L. Jensen, S. E. Rees, and J. G. Chase, "Simulation of Cardiovascular System Diseases by Including the Autonomic Nervous System in a Minimal Model," Comput Methods Programs Biomed, vol. 86(2), pp. 153-160, 2007.

[31] Smith B. W., J. G. Chase, G. M. Shaw, and R. I. Nokes, "Experimentally verified minimal cardiovascular system model for rapid diagnostic assistance," Control Engineering Practice, vol. 13, pp. 1183-1193, Sep 2005.

[32] Hann C., J. Chase, S. Andreassen, B. Smith, and G. Shaw, "Diagnosis Using a Minimal Cardiac Model Including Reflex Actions," Intensive Care Med, vol. 31, p. S18, 2005.

[33] Hann C., S. Andreassen, B. Smith, G. Shaw, and J. Chase, "Identification of Time-Varying Cardiac Disease State Using a Minimal Cardiac Model with Reflex Actions," in 14th IFAC Symposium on System Identification (SYSID 2006), Newcastle, Australia, 2006, pp. 475-480.

[34] Youssef I. K. and H. A. El-Arabawy, "Picard iteration algorithm combined with Gauss-Seidel technique for initial value problems," Applied Mathematics and Computation, vol. 190(1), pp. 345-355, 2007.

[35] Rao G. P. and H. Unbehauen, "Identification of continuous-time systems," IEE Proc. Control Theory Appl., vol. 153(2), pp. 185-220, 2006.

[36] Carson E. R. and C. Cobelli, Modelling methodology for physiology and medicine. San Diego: Academic Press, 2001.

[37] Muralidhar K., "Central venous pressure and pulmonary capillary wedge pressure monitoring," Indian J. Anaesth, vol. 46, pp. 298-303, 2002.

[38] Ghuysen A., B. Lambermont, P. Kolh, V. Tchana-Sato, D. Magis, P. Gerard, M. Mommens, N. Janssen, T. Desaive, and V. D'Orio, "Alteration of Right Ventricular-Pulmonary Vascular Coupling in a Porcine Model of Progressive Pressure Overloading," Shock, vol. 29(2), pp. 197-204, 2007.

[39] Ghuysen A., B. Lambermont, P. Kolh, V. Tchana-Sato, D. Magis, P. Gerard, M. Mommens, N. Janssen, T. Desaive, and V. D'Orio, "Alteration of Right Ventricular-Pulmonary Vascular Coupling in a Porcine Model of Proressive Pressure Overloading," Shock, vol. 29, pp. 197-204, 2007.

[40] Ueda H., T. Hayashi, K. Tsumura, K. Yoshimaru, Y. Nakayama, and J. Yoshikawa, "Inflection point of ascending aortic waveform is a predictive 
factor for all-cause and cardiovascular mortality in patients with chronic renal failure on hemodialysis," Am J Hypertens, vol. 17, pp. 1151-5, 2004.

[41] Mukkamala R. and R. J. Cohen, "A forward model-based validation of cardiovascular system identification," American Journal of Physiology. Heart and Circulatory Physiology, vol. 281(6), pp. H2714-2730, 2001.

[42] Kerckhoffs R. C., M. L. Neal, Q. Gu, J. B. Bassingthwaighte, J. H. Omens, and A. D. McCulloch, "Coupling of a 3D finite element model of cardiac ventricular mechanics to lumped systems models of the systemic and pulmonic circulation.," Ann Biomed Eng, vol. 35, pp. 1-18, 2006.

[43] Liang F. and H. Liu, "A Closed-Loop Lumped Parameter Computational Model for Human Cardiovascular System," JSME International Journal Series C, vol. 48, pp. 484-493, 2005.

[44] Mukkamala R., K. Toska, and R. J. Cohen, "Noninvasive identification of the total peripheral resistance baroreflex," Am J Physiol Heart Circ Physiol, vol. 284(3), pp. 947-959, 2003.

[45] Shim E. B., J. Y. Sah, and C. H. Youn, "Mathematical Modeling of Cardiovascular System Dynamics Using a Lumped Parameter Method," The Japanese Journal of Physiology, vol. 54(6), pp. 545-553, 2004.

[46] Senzaki H., C. H. Chen, and D. A. Kass, "Single-Beat Estimation of EndSystolic Pressure-Volume Relation in Humans A New Method With the Potential for Noninvasive Application," Circulation, vol. 94(10), pp. 24972506, 1996. 